\title{
Influencia de las infecciones helmínticas y el estado nutricional en la respuesta inmunitaria de niños venezolanos
}

\author{
Diana Ortiz, ${ }^{1}$ Celsa Afonso, ${ }^{2}$ Isabel Hagel, ${ }^{2}$ Orquídea Rodriguez, ${ }^{2}$ \\ Conchita Ortiz, ${ }^{3}$ Miguel Palenque ${ }^{4}$ y Neil R. Lynch ${ }^{4}$
}

RESUMEN El presente trabajo tuvo por objetivo estudiar la influencia del estado nutricional, determinado por medición antropométrica, y las infecciones helmínticas sobre la respuesta inmunitaria de niños de bajo nivel socioeconómico en dos poblaciones rurales diferentes de Venezuela: El Cardón, Estado Nueva Esparta, y San Daniel, Estado Miranda. Participaron en el estudio 125 niños de ambos sexos entre 2 y 15 años de edad, cuyo estrato socioeconómico se determinó por el método de Graffar modificado. Se les realizó un examen físico y una evaluación antropométrica tomando en cuenta los indicadores peso-talla, peso-edad, y talla-edad según los parámetros establecidos por la OMS. También se les practicaron exámenes de heces, IgA secretoria en saliva e IgE sérica total e inmunoglobulinas específicas anti-Ascaris. Ambas poblaciones pertenecían a los estratos IV y $V$ de la escala de Graffar, con un mayor número significativo $(\mathrm{P}<0,001)$ de habitantes de estrato $V$ en San Daniel. Los resultados sugieren que el grado de exposición y la susceptibilidad del individuo a los parásitos son factores que determinan las infecciones parasitarias y el comportamiento del sistema inmunitario. La intensidad de la carga parasitaria tiene una función importante en la estimulación de la IgE policlonal, lo que disminuye la efectividad de la respuesta específica para resistir esas infecciones. Por otra parte, las deficiencias nutricionales podrían alterar los mecanismos de inmunidad de las mucosas e influir negativamente en la síntesis de IgA secretoria y estimular la producción de IgE policlonal. Las condiciones ambientales y socioeconómicas deficientes favorecen una mayor exposición a los parásitos gastrointestinales y un estado nutricional deficiente, lo que modula la respuesta inmunitaria y afecta a los mecanismos de producción de la IgE sérica y la $\operatorname{Ig} A$ secretoria.

Los países en desarrollo están sufriendo un riguroso y sostenido dete-

\footnotetext{
Toda la correspondencia debe enviarse a esta autora, a la siguiente dirección: Instituto de Biomedicina, Universidad Central de Venezuela, San Nicolás a Providencia Parroquia San José (al lado del Hospital Vargas), Caracas, Venezuela, Zona postal 1010A. Apartado postal 4043. Correo electrónico: dprincz@hotmail.com

2 Instituto de Biomedicina, Universidad Central de Venezuela, Caracas, Venezuela.

3 Ministerio de Sanidad y Desarrollo Social, Caracas, Venezuela.

4 Clínica Experimental de Asma, Fondo de Desarrollo para el Estado Nueva Esparta, Estado Nueva Esparta, Venezuela.
}

rioro socioeconómico que se refleja en el estado de salud de la población y, principalmente, en el de los niños $(1,2)$. Como es bien sabido, el nivel socioeconómico está estrechamente relacionado con la prevalencia de infecciones por parásitos intestinales $(1,3,4)$. Además, la falta de recursos para adquirir alimentos trae como consecuencia deficiencias en la alimentación de los niños de amplios sectores de la población. Aunque en casi todos los países de América Latina y el Caribe ha disminuido la desnutrición grave, existe un estado crónico de subalimentación que produce altas tasas de desnutrición leve y moderada (5). Por otro lado, se ha comprobado que hay una relación entre las infecciones parasitarias y la malnutrición. Por ejemplo, las infecciones por parásitos intestinales comunes tales como Ascaris lumbricoides, Trichuris trichiura y Giardia lamblia pueden acelerar el tránsito intestinal y alterar el equilibrio de nitrógeno por excesiva pérdida de este elemento en las heces, lo que produce malabsorción e intolerancia a azúcares y vitaminas 
(6-9). En el medio clínico se ha informado de que las infecciones helmínticas pueden producir malnutrición proteinocalórica y que los niños preescolares son los más vulnerables a este tipo de desnutrición (6-9). Por lo tanto, las infecciones pueden tener un efecto negativo sobre la rapidez del crecimiento en los niños afectados $(9,10)$.

Estas deficiencias nutricionales podrían, a su vez, influir en el estado de infección parasitaria por medio de la modulación de la respuesta inmunitaria $(2,11)$, que está involucrada en los mecanismos de defensa contra los parásitos (12). Por ejemplo, la malnutrición proteinocalórica influye en la producción de IgA secretoria, (8) que cumple un papel importante en la inmunidad de la mucosa gastrointestinal $(8,13)$. Por otra parte, la IgE desempeña una función importante en los mecanismos de protección contra las infecciones helmínticas $(12,14-16)$ y esa respuesta es influida por el estado nutricional (12). Se ha determinado que los helmintos no solo estimulan respuestas específicas de la IgE, sino que además provocan una síntesis policlonal de la IgE inducida por los linfocitos ayudantes tipo 2 y la interleuquina 4 (Th2/IL4) $(15,17,18)$. Esa respuesta policlonal podría representar un mecanismo de los parásitos para evadir la respuesta inmunitaria (15). Ya que el estado de deficiencia nutricional favorece la policlonalidad, es posible que contribuya así a un aumento de la infección parasitaria (12).

El objetivo del presente trabajo fue estudiar la relación entre los factores ambientales y la intensidad de la infección parasitaria y entre el estado nutricional y la respuesta humoral a los helmintos intestinales $A$. lumbricoides y $T$. trichiura en niños de dos poblaciones rurales.

\section{MATERIALES Y MÉTODOS}

La muestra de estudio consistió en 125 niños de dos comunidades rurales de bajo nivel socioeconómico que se diferenciaban en cuanto a la principal actividad económica de la población de origen y las características ecológi- cas locales. La población de San Daniel, ubicada en el Municipio Guaicaipuro del Estado Miranda, trabajaba en actividades agrícolas en un clima tropical lluvioso. La población de El Cardón de la Isla de Coche, Estado Nueva Esparta, se ocupaba principalmente de la pesquería en un clima seco, de vegetación xerófila.

En el estudio se incluyeron todos los niños de las escuelas pertenecientes a El Cardón y San Daniel cuyos guardianes legales autorizaron su participación por escrito. La muestra de niños de ambos sexos comprendió 43 preescolares de 1 a 6 años de edad, 75 escolares de 7 a 12 años y 7 preadolescentes de 13 a 15 años. En cuanto a origen local, 50 participantes eran de El Cardón (21 niñas y 29 niños) con una media de edad de $8,1 \pm 2,6$, y 75 de San Daniel (39 niñas y 36 niños) con una media de edad de $7,3 \pm 3,8$.

El protocolo de estudio fue aprobado por la comisión ética del Instituto de Biomedicina, Facultad de Medicina de la Universidad Central de Venezuela, y por las autoridades competentes de los centros rurales de consulta externa donde se llevó a cabo. La investigación se desarrolló durante el período de enero de 1995 a septiembre de 1997.

Además de evaluar a cada niño por medio de un examen físico, se obtuvo información complementaria mediante un interrogatorio de los padres o guardianes. Médicos de los centros de salud de las comunidades de El Cardón y San Daniel realizaron los exámenes físicos.

También se evaluó el nivel socioeconómico de la familia de cada niño participante por el método de Graffar modificado para Venezuela por $\mathrm{H}$. Méndez Castellano (19). De acuerdo con el método, se tomaron en cuenta la ocupación del jefe de familia, el grado de instrucción de la madre, las fuentes de ingreso y las condiciones de la vivienda. Además, se consideraron factores sanitarios como la disposición de excretas y de basura, y la disponibilidad de agua.

En el análisis antropométrico se determinaron las relaciones peso-talla, talla-edad y peso-edad según las curvas de crecimiento elaboradas por el
Instituto Nacional de Nutrición de Venezuela con los valores publicados por la OMS (20). Los niños se clasificaron en adecuadamente nutridos o con deficiencia nutricional - leve, moderada o grave- de acuerdo con los parámetros propuestos en las bases para el desarrollo de guías de alimentación del Instituto Nacional de Nutrición, que se muestran en el anexo 1. El estado nutricional se definió por la presencia del mismo parámetro (deficiencia grave, moderada o leve, o nutrición adecuada) en un mínimo de dos indicadores antropométricos, dándole prioridad a la talla.

La evaluación parasitológica se basó en los exámenes de las heces por el método de Kato-Katz para determinar la presencia y el número de huevos de helmintos por gramo de heces.

Para determinar la concentración total de IgA secretoria en la saliva, esta se recolectó directamente de la boca de cada niño. Las muestras se conservaron en tubos Vacutainer con ácido etilendiaminatetraacético (EDTA) a $-20^{\circ} \mathrm{C}$ hasta su uso en el ensayo. Se empleó la técnica ELISA en placas de microtitulación de poliestireno (DYNATECH). Estas se sensibilizaron con un anticuerpo monoclonal anti-IgA humano (cadena $\alpha$ específica: SIGMA) diluido en amortiguador a 1:50. Como solución de bloqueo se utilizó suero de caballo diluido al $10 \%$ en PBS y las muestras de saliva se agregaron a las placas en una dilución de 1:1 000 en PBS-suero de caballo. Luego se añadió una anti-IgA marcada con peroxidasa a una dilución de 1:4 000 en PBS-suero de caballo y finalmente se revelaron los resultados con ortofenilenodiamina (OPD) (SIGMA) leyendo la absorbancia a $492 \mathrm{~nm}$.

La IgA secretoria anti-Ascaris específica se determinó en placas flexibles de polivinilo (FALCON) sensibilizadas durante toda la noche a $4{ }^{\circ} \mathrm{C}$ con un extracto de Ascaris en concentración de $3 \mathrm{mg} / \mathrm{mL}$ diluidos a 1:30 en PBS. Las placas fueron bloqueadas con suero de caballo diluido a 1:30 en PBS. Las muestras de saliva se diluyeron a 1:10 en PBS con suero de caballo 1:30. Para el conjugado se utilizó anti-IgA marcada con peroxidasa a una dilución de 1:1 000 en PBS-suero de caballo. Se re- 
veló con OPD y se leyó la absorbancia a $492 \mathrm{~nm}$.

El antígeno de Ascaris se obtuvo mediante purificación parcial de un extracto liofilizado del cuerpo completo del helminto, para lo cual se disolvió $1 \mathrm{~g}$ de cuerpo completo de $A$. lumbricoides liofilizado en $100 \mathrm{~mL}$ de agua y se centrifugó. Se guardó el sobrenadante y se resuspendió el precipitado en $2 \mathrm{~mL}$ de $\mathrm{KCl} 3 \mathrm{~mol} / \mathrm{L}$, que se mantuvo en agitación durante toda la noche a $4{ }^{\circ} \mathrm{C}$. El sobrenadante obtenido de esta forma se mezcló con el de la primera centrifugación y la mezcla se precipitó a $85 \%$ de saturación en sulfato de amonio y se centrifugó. El precipitado resultante se resuspendió en $2,5 \mathrm{~mL}$ de agua bidestilada. Se dializó en $2 \mathrm{~L}$ de agua bidestilada con fenol a $0,04 \%$ con agitación continua a $4{ }^{\circ} \mathrm{C}$. Luego se midió la concentración de proteínas por el método de Bradford.

Para precisar la IgE total en suero, se utilizaron como fase sólida del ensayo placas de microtitulación de polivinilo sensibilizadas con IgE monoclonal (2.1.5 elaborada en Australia) añadida a $1 \mu \mathrm{g} /$ pozo diluido en amortiguador con un $\mathrm{pH}$ de 9,6. Como solución de bloqueo se empleó PBS Tween-BSA a $1 \%$ y las muestras se diluyeron en esa solución a razón de 1:400. Se utilizaron como referencia diluciones de 1:400, 1:1 000, 1:2 000, 1:8 000 y 1:32 000 de un conjunto de sueros con altas concentraciones de IgE total, que fueron estandarizados con la prueba comercial Phadebas IgE Paper Inmuno Sorbent Test (PRIST) de Pharmacia, Suecia, y evaluados en un laboratorio de referencia. Luego se agregó una $\alpha \operatorname{IgE}$ biotinilada comercial (Vector) a una dilución de 1:500 en PBS Tween-BSA y, por último, para amplificar la reacción, se agregaron $50 \mu \mathrm{L} /$ pozo de avidinabiotina diluida en PBS Tween-BSA a $1 \%$. Para ello se utilizó el estuche de Vector-Vectastain. Se reveló con OPD y se leyó la absorbancia a $492 \mathrm{~nm}$.

Para perfilar la IgE sérica específica, se utilizaron como sensibilizador 3 $\mathrm{mg} / \mathrm{mL}$ del antígeno de Ascaris diluido en amortiguador con un $\mathrm{pH}$ de 9,6 y, como solución de bloqueo, la PBSTween-BSA a 1\%. Se formó un pool de sueros cuyas concentraciones de IgE específica pertenecían al Radio Allergo Sorbent Test (RAST) clase 4; es decir, eran mayores que $17,5 \mu \mathrm{L} / \mathrm{mL}$, y para elaborar la curva de referencia se emplearon diluciones del pool de 1:10, 1:20, 1:50, 1:100, 1:200 y 1:400. Luego se agregó el anticuerpo monoclonal anti-IgE (2.1.5 preparado en Australia) a una concentración de $1 \mu \mathrm{g} /$ pozo, y posteriormente se añadió una anti-IgG de ratón marcada con peroxidasa a una dilución de 1:3 000 en PBS-Tween-BSA a $1 \%$, se reveló la reacción con OPD y, por último, se leyó la absorbancia a 492 nm. Se determinó además el índice de policlonalidad tomando en cuenta la relación de $\operatorname{IgE}$ total/IgE específica.

En el análisis estadístico, los valores de IgE total e IgE específicos se transformaron a logaritmos, debido a que no tienen una distribución normal, y se calcularon las medias y las desviaciones estándar. También se calcularon las medias y las desviaciones estándar de los valores de IgA secretoria total y específica, que fueron expresados en unidades de densidad óptica. Las comparaciones entre los diferentes grupos se realizaron mediante la prueba $t$ de Student y las diferencias entre proporciones se compararon aplicando la prueba de ji al cuadrado $\left(\chi^{2}\right)$.

\section{RESULTADOS}

Desde el punto de vista socioeconómico, los resultados indican (cuadro 1) que la mayoría de la población muestral pertenecía a los estratos IV y V de la escala de Graffar, y que en San Daniel predominaba significativamente el estrato $\mathrm{V}(P<0,001)$. En la población de El Cardón, $82 \%$ de las madres de los niños estudiados tenían algún grado de instrucción, la mayoría (56\%) a nivel de primaria. Por el contrario, $66 \%$ de las madres correspondientes en la población de San Daniel resultaron ser analfabetas y, del $34 \%$ que tenían algún grado de escolaridad, la mayoría era primaria.
CUADRO 1. Comparación socioeconómica de las familias de 125 niños estudiados en dos poblaciones rurales de Venezuela

\begin{tabular}{|c|c|c|}
\hline \multirow[b]{2}{*}{ Característica } & \multicolumn{2}{|c|}{ Población (\%) } \\
\hline & $\begin{array}{l}\text { El Cardón } \\
\quad(n=27)\end{array}$ & $\begin{array}{c}\text { San Daniel } \\
(n=34)\end{array}$ \\
\hline \multicolumn{3}{|l|}{ Nivel de Graffar } \\
\hline III & 4 & 0 \\
\hline IV & 37 & 9 \\
\hline V & 59 & 91 \\
\hline \multicolumn{3}{|l|}{ Grado de instrucción de la madre } \\
\hline Secundaria completa & 6 & 0 \\
\hline Secundaria incompleta & 19 & 7 \\
\hline Primaria & 56 & 27 \\
\hline Analfabeta & 19 & 66 \\
\hline \multicolumn{3}{|l|}{ Condiciones de vivienda } \\
\hline Buenas condiciones sanitarias & 4 & 0 \\
\hline Deficiencia en algunas condiciones & & \\
\hline sanitarias & 77 & 26 \\
\hline Deficiencia en todas las condiciones & & \\
\hline sanitarias & 19 & 74 \\
\hline \multicolumn{3}{|l|}{ Disposición de excretas } \\
\hline Al aire libre & 4 & 43 \\
\hline En pozo séptico & 96 & 57 \\
\hline \multicolumn{3}{|l|}{ Disposición de la basura } \\
\hline Aseo urbano (2 ó 3 veces por & & \\
\hline semana) & 100 & 0 \\
\hline Quema & 0 & \\
\hline Disponibilidad semanal de agua & 29 & 100 \\
\hline
\end{tabular}


Con respecto a las condiciones de vivienda en la población de El Cardón, $77 \%$ de los hogares de los niños estudiados eran deficientes en algunos aspectos sanitarios. En la población de San Daniel, 74\% de las viviendas presentaron deficiencias similares.

Por otra parte, en la población de El Cardón 96\% de las viviendas tenían pozo séptico y solo en $4 \%$ se disponía de las excretas al aire libre. Sin embargo, en San Daniel 57\% de la población estudiada disponía de las excretas al aire libre, mientras que $43 \%$ tenían pozo séptico en la vivienda.

Con respecto a la disposición de basura, la población de El Cardón contaba con el servicio de aseo urbano que la recogía dos o tres veces por semana, mientras que en San Daniel toda la población quemaba la basura. Además, en El Cardón se recibía agua a domicilio solo dos o tres veces por semana, mientras que en San Daniel se recibía agua continuamente.

En lo que se refiere a las parasitosis helmínticas por Ascaris y Trichuris, la prevalencia en la población de El Cardón fue significativamente mayor $(P<0,0001)$ que en la de San Daniel (cuadro 2). En El Cardón, 70\% de los niños estudiados estaban parasitados con Trichuris en comparación con $22 \%$ de los de San Daniel $(P<0,05)$. En San Daniel, 30\% de los niños estaban parasitados con Ascaris y en El Cardón, 38\%.

Sin embargo, aunque en la población de El Cardón encontramos una mayor prevalencia de parasitosis helmínticas, la carga parasitaria de Ascaris y Trichuris fue significativamente mayor $(P<0,05)$ en San Daniel (cuadro 2$)$.

En ambas poblaciones hubo altos porcentajes de niños con deficiencias en relación con el indicador talla-edad (74\% en San Daniel y $44 \%$ en El Cardón). Le siguieron en importancia las deficiencias en peso-edad (65\% en San Daniel y $42 \%$ en El Cardón) y, por último, las del indicador peso-talla (39\% en San Daniel y 20\% en El Cardón) (cuadro 3). En San Daniel hubo un porcentaje significativamente mayor $(P<0,05)$ de niños bajo la norma de los

CUADRO 2. Media más desviación estándar de la intensidad de carga parasitaria en dos poblaciones rurales de niños venezolanos, expresada en el número de huevos por gramo de heces, según el método de Kato/Katz

\begin{tabular}{|c|c|c|c|}
\hline Población & $\begin{array}{c}\text { Total de } \\
\text { helmintos }\end{array}$ & Ascaris & Trichuris \\
\hline $\begin{array}{c}\text { El Cardón } \\
n=50\end{array}$ & $\begin{array}{c}80 \% \%^{\mathrm{a}} \\
(1080 \pm 609)\end{array}$ & $\begin{array}{c}38 \% \%^{b} \\
(2072 \pm 1650)\end{array}$ & $\begin{array}{c}70 \%{ }^{\mathrm{c}} \\
(324 \pm 156)\end{array}$ \\
\hline $\begin{array}{c}\text { San Daniel } \\
n=75\end{array}$ & $\begin{array}{c}35 \% \\
(2766 \pm 1944)\end{array}$ & $\begin{array}{c}30 \% \\
(2797 \pm 1831)\end{array}$ & $\begin{array}{c}22 \% \\
(510 \pm 315)\end{array}$ \\
\hline
\end{tabular}

CUADRO 3. Porcentajes de niños con indicadores antropométricos adecuados y bajo la norma en dos poblaciones rurales de Venezuela

\begin{tabular}{|c|c|c|c|c|c|c|}
\hline \multirow[b]{2}{*}{ Población } & \multicolumn{2}{|c|}{ Peso-edad } & \multicolumn{2}{|c|}{ Peso-talla } & \multicolumn{2}{|c|}{ Talla-edad } \\
\hline & Adecuado & $\begin{array}{l}\text { Bajo la } \\
\text { norma }\end{array}$ & Adecuado & $\begin{array}{l}\text { Bajo la } \\
\text { norma }\end{array}$ & Adecuada & $\begin{array}{l}\text { Bajo la } \\
\text { norma }\end{array}$ \\
\hline $\begin{array}{c}\text { El Cardón } \\
n=50\end{array}$ & $58^{a}$ & $42^{a}$ & $80^{a}$ & $20^{\mathrm{a}}$ & $56^{a}$ & $44^{a}$ \\
\hline $\begin{array}{c}\text { San Daniel } \\
n=75\end{array}$ & 35 & 65 & 60 & 39 & 25 & 74 \\
\hline
\end{tabular}

tres indicadores antropométricos que en El Cardón (véase el cuadro 3).

En las dos poblaciones, los indicadores antropométricos señalaron también altas proporciones de niños con deficiencias nutricionales (cuadro 4). En la población de San Daniel, 51\% de los niños estudiados presentaron deficiencias nutricionales leves y $23 \%$, deficiencias moderadas, o sea, que $74 \%$ de los niños estaban bajo la norma. En El Cardón, 63\% de los niños estudiados presentaron deficiencias nutricionales: $53 \%$ deficiencias leves y 10\%, moderadas. Como es de notar, en San Daniel la desnutrición era más intensa, con predominio de la forma moderada.

En relación con el estado inmunitario de los participantes, se determinó que las concentraciones de IgA secretoria total en saliva en la población de El Cardón fueron significativamente mayores $(P<0,001)$ que en San Daniel. Em cambio, la IgE sérica total en San Daniel fue significativamente mayor $(P<0,001)$ que en El Cardón (cuadro 5).

Las concentraciones de IgA secretoria específica anti-Ascaris en saliva fueron significativamente mayores $(P<0,001)$ en San Daniel que en El Cardón, mientras que las concentraciones séricas de IgE específica contra Ascaris fueron similares en ambas poblaciones. Además, el índice de policlonalidad fue significativamente mayor $(P<0,05)$ en San Daniel que en el Cardón (véase el cuadro 5).

Observamos además que en El Cardón, población donde los niveles de IgA secretoria total fueron más elevados, la $\operatorname{Ig}$ A secretoria específica fue significativamente menor $(P<0,001)$ que en San Daniel, donde la IgA secretoria total fue menor.

Agrupando las dos poblaciones de niños que presentaron deficiencias nutricionales (figura 1), observamos que hay una tendencia a la disminución de la $\operatorname{Ig}$ A secretoria total a medida que empeora el estado nutricional. Además, encontramos que la IgE total aumenta significativamente $(P<0,001)$ en niños con deficiencias nutricionales (figura 2). No se encontró ninguna diferencia entre los grupos con respecto a la producción de IgA secretoria específica anti-Ascaris (figura 3). Sin embargo, notamos además que la $\operatorname{IgE}$ específica 
CUADRO 4. Porcentajes de niños con deficiencias nutricionales de acuerdo con los parámetros antropométricos establecidos para el estudio

\begin{tabular}{lccc}
\hline Población & $\begin{array}{c}\text { Alguna deficiencia } \\
\text { nutricional }\end{array}$ & $\begin{array}{c}\text { Deficiencia } \\
\text { leve }\end{array}$ & $\begin{array}{c}\text { Deficiencia } \\
\text { moderada }\end{array}$ \\
\hline $\begin{array}{c}\text { El Cardón } \\
n=50\end{array}$ & 63 & 53 & 10 \\
$\begin{array}{c}\text { San Daniel } \\
n=75\end{array}$ & 75 & 51 & 23 \\
\hline
\end{tabular}

CUADRO 5. Concentraciones de inmunoglobulinas totales y especificas anti-Ascaris e índice de policlonalidad en dos poblaciones rurales de niños venezolanos

\begin{tabular}{|c|c|c|}
\hline \multirow[b]{2}{*}{ Inmunoglobulina y policlonalidad } & \multicolumn{2}{|c|}{ Población } \\
\hline & El Cardón & San Daniel \\
\hline $\begin{array}{l}\text { IgA secretoria total } \\
\left(^{(U D O)^{a}}\right.\end{array}$ & $0,80 \pm 0,25$ & $0,23 \pm 0,15^{b}$ \\
\hline $\begin{array}{l}\operatorname{Ig} \text { A secretoria } \\
\text { específica anti- } \\
\text { Ascaris (UDO) }\end{array}$ & $0,32 \pm 0,24$ & $0,44 \pm 0,18^{b}$ \\
\hline $\begin{array}{l}\text { IgE sérica total } \\
(\mathrm{Ul} / \mathrm{mL})^{c}\end{array}$ & $\begin{array}{c}1202 \\
(1382)\end{array}$ & $\begin{array}{l}1778^{b} \\
(1998)\end{array}$ \\
\hline $\begin{array}{l}\text { IgE específica anti-Ascaris } \\
\quad(\mathrm{Ul} / \mathrm{mL})^{\mathrm{a}}\end{array}$ & $0,84 \pm 0,23$ & $0,85 \pm 0,28$ \\
\hline $\begin{array}{l}\text { Índice de } \\
\text { policlonalidad }\end{array}$ & $1431 \pm 480$ & $2092 \pm 512$ \\
\hline
\end{tabular}

a Media \pm desviación estándar; UDO = unidades de densidad óptica.

b $P<0,001$.

c Media y media más 1 desviación estándar, calculadas por transformación logarítmica.

tiene un comportamiento contrario a la IgE total, de manera que disminuye notablemente a medida que empeora el estado nutricional (figura 4).

\section{DISCUSIÓN}

Los resultados de la parte socioeconómica del estudio indican que en la población de El Cardón existían grandes deficiencias en cuanto a disponibilidad de agua. La disposición de basura era inadecuada, lo que limitaba las condiciones de higiene aun cuando la disposición de excretas era adecuada. En cambio, en San Daniel no había problemas con la disponibilidad de agua y tampoco con la disposición de basura, pero la disposición de excretas era inadecuada.

Estas observaciones son importantes, ya que en la mayoría de los estudios anteriores se ha encontrado una relación inversa entre la adecuada disposición de excretas y la incidencia de parasitosis intestinales $(5,15)$. Cabe inferir que la alta prevalencia de parasitosis helmínticas encontrada en El Cardón se debe seguramente a las condiciones ambientales desfavorables particulares en las que se encuentra la población, ya que la poca disponibilidad de agua y su almacenamiento inadecuado, así como las deficientes condiciones higiénicas de la vivienda, favorecen la exposición a los helmintos. Sin embargo, a pesar de que había más contacto con los pará-
FIGURA 1. Concentraciones de IgA secretoria total de acuerdo con el estado nutricional en grupos combinados de dos poblaciones rurales de niños venezolanos

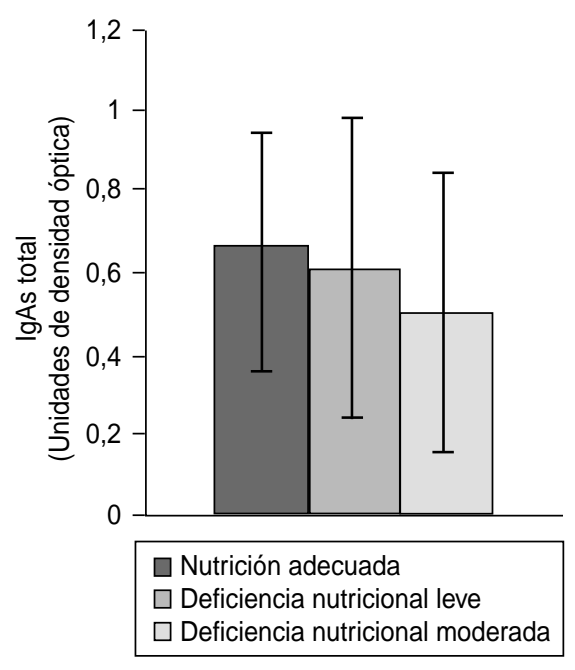

FIGURA 2. Concentraciones de IgE total de acuerdo con el estado nutricional en grupos combinados de dos poblaciones rurales de niños venezolanos

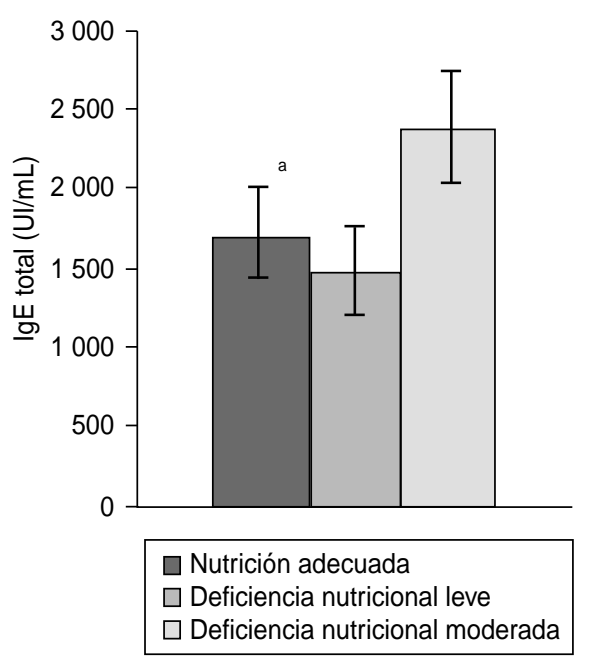

${ }^{\mathrm{a}} P<0,001$.

sitos en El Cardón, se encontró una carga parasitaria más intensa en la población de San Daniel, lo que puede reflejar características intrínsecas de la población que quizá aumenten la susceptibilidad de los niños a las infecciones parasitarias. 
FIGURA 3. Concentraciones de IgA secretoria específica anti-Ascaris de acuerdo con el estado nutricional en grupos combinados de dos poblaciones rurales de niños venezolanos

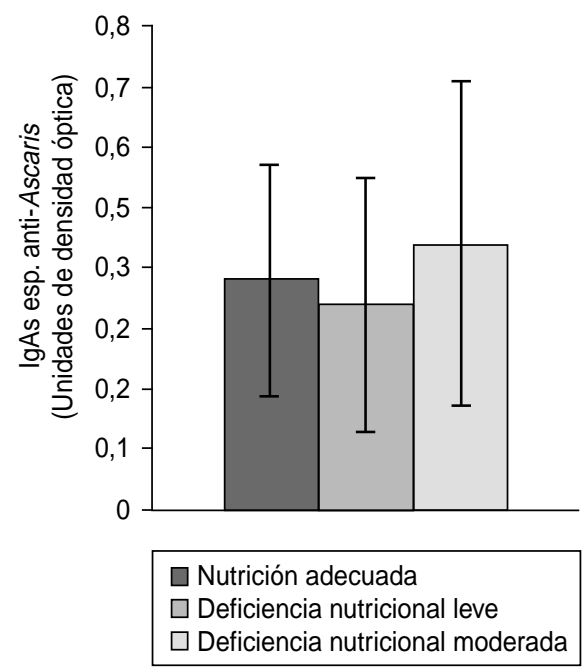

FIGURA 4. Concentraciones de IgE específica anti-Ascaris de acuerdo con el estado nutricional en grupos combinados de dos poblaciones rurales de niños venezolanos

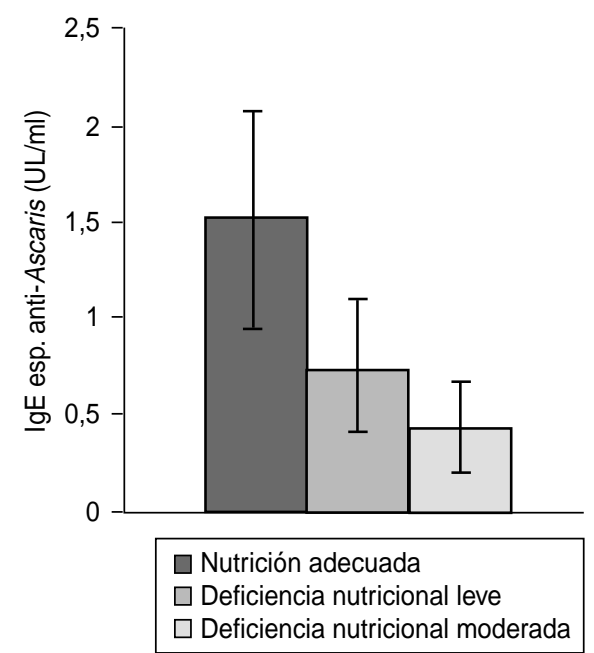

Es importante señalar que hubo una mayor proporción de niños con deficiencias nutricionales en la población de San Daniel que en la de El Cardón, lo que se reflejó en los tres indicadores antropométricos de peso-talla, tallaedad y peso-edad y que, frente a esta diferencia, los niños con deficiencias nutricionales moderadas fueron los más perjudicados. Teniendo en cuenta que en la población de San Daniel la dieta está basada principalmente en el consumo de carbohidratos, especialmente pastas, frijol negro y harina precocida de maíz (datos no presentados), y que la ingesta de alimentos de origen animal es bastante limitada, es posible que existan deficiencias de vitamina A, complejo vitamínico B, hierro y cinc. La población de El Cardón, a pesar de ser igualmente de bajo nivel socioeconómico, por sus características geográficas y ambientales tiene acceso al pescado, fuente importante de proteínas animales, hierro, cinc y fósforo. Las deficiencias mencionadas pueden influir desfavorablemente en la respuesta inmunitaria de la población de San Daniel, según indican los conocimientos actuales sobre la importancia de los oligoelementos y las vitaminas en las funciones del sistema inmunitario, y la predisposición del niño malnutrido a las infecciones $(7,21)$. Esto podría influir en la elevada carga parasitaria encontrada en la población de San Daniel, ya que se ha comprobado que el estado nutricional influye en la resistencia a las infecciones por parásitos (22).

Entre los mecanismos antiparasitarios del sistema inmunitario existen dos factores importantes: las respuestas de la IgE y de la IgA secretoria. Las concentraciones de la IgA secretoria total fueron significativamente más altas $(P<0,001)$ en la población de El Cardón que en la de San Daniel. Teniendo en cuenta que en El Cardón la proporción de niños con deficiencias nutricionales fue menor que en San Daniel —población donde se encontró un mayor número de niños con deficiencias nutricionales moderadas-, puede inferirse que las concentraciones de IgA secretoria total están directamente relacionadas con el estado nutricional. Diversas pruebas clínicas y modelos experimentales sugieren que la malnutrición altera los mecanismos de defensa de la inmunidad de las mucosas y compromete la morfología y la función de la barrera intestinal contra la invasión de microorganismos y agentes patógenos en general (8). Es posible que en niños con malnutrición proteinocalórica exista una falla de la síntesis de la IgA o bien un bloqueo en el transporte de esta a las superficies mucosas (13).

Sin embargo, los valores de la IgA secretoria específica anti-Ascaris fueron significativamente más elevados $(P<0,001)$ en San Daniel, lo que se refleja además en una relación inversa entre la producción de la IgA secretoria total y la IgA secretoria específica. En este aspecto podrían influir alteraciones de la mucosa gastrointestinal ocasionadas por el deterioro nutricional o bien algún problema en la maduración de la afinidad de los anticuerpos. Se provocaría así un descontrol que podría relacionarse igualmente con las infecciones helmínticas de menor prevalencia pero elevada intensidad en esa población. En este sentido, es importante señalar que sería muy útil establecer los mecanismos involucrados en la producción de IgA secretoria para determinar si las concentraciones de la IgA secretoria total y la específica pueden utilizarse como método de vigilancia epidemiológica para detectar a los niños en riesgo nutricional. Este aspecto sigue siendo de especial interés para nuestro grupo de investigación.

Aunque los factores ambientales asociados a la malnutrición y las parasitosis son similares, los niños con deficiencias nutricionales presentan una menor prevalencia de parasitosis, comparados con aquellos que están nutridos adecuadamente. Esto podría explicarse en función de un microambiente intestinal deficiente para el parásito en una situación de déficit nutricional.

Encontramos asimismo que las concentraciones de IgE total en la población de San Daniel fueron significativamente mayores $(P<0,001)$ que las de El Cardón. Quizá ello refleje las altas cargas parasitarias, ya que la intensidad parasitaria es un factor importante en la estimulación de la $\operatorname{IgE}$ policlonal, que disminuye la efectividad de la respuesta específica involucrada en la resistencia a estas infecciones. Además, podrían estar influyendo otros parásitos no incluidos en el estudio. De acuerdo con estos resultados, en trabajos anteriores de nuestro grupo 
hemos encontrado que los bajos niveles de IgE específica anti-Ascaris predisponen al niño a un mayor riesgo de infección, al contrario de lo que ocurre en niños con valores elevados de $\operatorname{IgE}$ específica (12).

De esta manera, aunque los valores de IgE total fueron más elevados en San Daniel que en El Cardón, no se encontraron diferencias entre los niveles de IgE específica anti-Ascaris en ambas poblaciones, de manera que no hubo un aumento proporcional entre la producción de IgE total/IgE específica, por lo que se tomó en cuenta esta relación encontrando que el índice de policlonalidad fue significativamente mayor en San Daniel que en El Cardón. Esto sugiere que en El Cardón existe la capacidad de establecer una respuesta protectora más eficiente, lo que se relaciona, además, con el hecho de que en esta población existen menos niños con deficiencias nutricionales. En efecto, la IgE total aumenta a medida que empeora el estado nutricional, lo que implica mayor policlonalidad, menor especificidad de la respuesta $\mathrm{y}$, por lo tanto, menor resistencia frente al parásito. Estas deficiencias nutricionales se asocian con frecuencia a cambios tisu- lares que contribuyen a la disminución de la resistencia del huésped contra la infección (13).

Un aspecto importante es que en estudios previos se ha determinado una alta incidencia de atopia en la población de El Cardón y se ha demostrado la capacidad intrínseca que tienen los niños atópicos de producir $\operatorname{IgE}$ frente a antígenos específicos. Presentan de esta manera una respuesta específica más efectiva frente al parásito $(9,23)$. Esta condición podría estimular respuestas IgE más eficientes y posiblemente de mayor afinidad, que limiten la policlonalidad característica de la respuesta frente a los helmintos normalmente observada en poblaciones rurales donde las infecciones helmínticas son muy frecuentes (9).

Encontramos también una relación inversa entre la producción de IgA secretoria total y la IgE sérica total. Es posible que en niños deficientes de IgA secretoria existan alteraciones de la homeostasia de la mucosa que favorezcan la sensibilización del sistema humoral por antígenos que estimulan la respuesta IgE. Esta podría estar ejerciendo un papel importante como protectora contra los helmintos.

\section{REFERENCIAS}

1. Cooper E. Intestinal parasitosis and the modern description of diseases of poverty. Trans $\mathrm{R}$ Soc Trop Med Hyg 1991;85:168-170.

2. Cunningham $S$. Nutrient modulation of the immune response. New York: Dekker; 1993.

3. Bundy DA. The global burden of intestinal nematode disease. Trans R Soc Trop Med Hyg 1994;88:259-261.

4. Jarret EE, Miller HRP. Productions and activities of IgE in helminth infection. Prog Allergy 1982;31:178-233.

5. Méndez Castellano H, López M, Benahím C, Maza Zavala D, González I. Estudio nacional de crecimiento y desarrollo humanos de la República de Venezuela, tomo III. Caracas: Ministerio de la Secretaría de la República de Venezuela, Centro de Estudios Biológicos para el Crecimiento y Desarrollo de Poblaciones de Venezuela (FUNDACREDESA); 1996. Pp. 1011-1018.

6. Crompton DW. Ascariasis and childhood malnutrition. Trans R Soc Trop Med Hyg 1992; 6:577-579.

7. Feigin RD, Garg R. Interacción entre infección y nutrición. En: Feigin RD, Cherry J, eds.
Tratado de infecciones en pediatría, vol 1. México, D.F.: Editorial Interamericana McGraw Hill; 1992. Pp. 15-24.

8. Welsh FKS, Farmery K, Lennan MAC, Sheridan MB, Barclay GR, Guillou PJ, et al. Gut barrier function in malnourished patients. Gut 1998;42:396-401.

9. Nesheim MC. Human nutrition needs and parasitic infection. Parasitology 1993;Suppl 107:S7-S17.

10. Marcano A, Ruiz F, Aldana M, Garasini M. Absceso hepático ascaridiano. Gen (Revista de la Sociedad Venezolana de Gastroenterología) 1987; 9(2):112-115.

11. Haswell-Elkins MR, Leonard H, Kennedy MW, Elkins DB, Maizels RM. Inmunoepidemiology of Ascaris lumbricoides: relationships between antibody specificities, exposure and infection in a human community. Parasitology 1992:104 Pt 1:153-159.

12. Hagel I, Lynch NR, Perez M, Di Prisco MC, Lopez R, Rojas E. Relationship between the degree of poverty and the IgE response to $\mathrm{As}^{-}$ caris infection in slum children. Trans $\mathrm{R}$ Soc Trop Med Hyg 1993;87(1):16-18.
Los factores socioeconómicos y ambientales son de gran importancia como elementos que influyen sobre los problemas nutricionales, parasitológicos e inmunológicos del individuo. Las condiciones ambientales deficientes favorecen una mayor exposición a los parásitos gastrointestinales. Se modula de esta manera la respuesta inmunitaria afectando así a los mecanismos de producción de algunas inmunoglobulinas tales como la IgE y la IgA secretoria. Por otra parte, algunas características intrínsecas de la población tales como su constitución genética e inmunológica podrían desempeñar una función importante en la susceptibilidad del individuo frente a las infecciones parasitarias. La exposición y la susceptibilidad parecen ser factores determinantes en las infecciones parasitarias y en el comportamiento del sistema inmunitario del individuo.

Agradecimiento. Este trabajo fue financiado por el Banco Mundial, proyecto VEN/096/002/014, y se hizo posible gracias a la colaboración de las comunidades donde se realizó la investigación y al personal del Instituto de Biomedicina.
13. Abbas A, Lichtman A, Pober J. Inmunología celular y molecular. 2a ed. Madrid: Interamericana McGraw Hill; 1995: 517.

14. Dunne DW, Butterworth AE, Fulford AJ, Kariuki HC, Langley JG, Ouma JH, et al. Immunity after treatment of human schistosomiasis: association between $\operatorname{IgE}$ antibodies to adult worm antigens and resistance to reinfection. Eur J Inmunol 1992; 22:1483-1494.

15. Lynch NR, Hagel I, Palenque M, Di Prisco MC, Escudero J, Corao LA, et al. Relationship between helminthic infection and IgE response in atopic and nonatopic children in a tropical environment. J Allergy Clin Immunol 1998;101:217-221.

16. Lee $\mathrm{TD}, \mathrm{Xie} \mathrm{CY}$. IgE regulation by nematodes: the body fluid of Ascaris contains a B-cell mitogen. J Allergy Clin Inmunol 1995;95(6): 1246-1254.

17. Finkelman F, Pearce E, Urban J, Sher A. Regulation and biological function of helminthicinduced cytokine responses. Immunol Today 1991;12:A62-66.

18. Hagel I, Lynch NR, Perez M., Di Prisco MC, Lopez R, Rojas E. Modulation of the allergic 
reactivity of slum children by helminthic infection. Parasite Immunol 1993;15:311-315.

19. Méndez Castellano H. Estratificación social y biología humana. Archivos Venezolanos de Puericultura y Pediatría 1986;49:93-104.

20. López Blanco M, Landaeta M. Crecimiento y desarrollo, nutrición y adolescencia. En: Sociedad Venezolana de Puericultura y Pediatría. Manual de crecimiento y desarrollo. Caracas: Centro de Estudios Biológicos para el Crecimiento y Desarrollo de Poblaciones de Venezuela (FUNDACREDESA); 1991.
21. Chandra RK. Parasite infection, nutrition and immune response. Fed Proc 1984;43(2): 251-255.

22. Díaz AI, Morales G, Pino L, Chourio LG Calchi M. Eficacia comparada de técnicas coproscópicas empleadas en la determinación de la intensidad geohelmíntica. Kasmera (revista de la Escuela de Medicina, Universidad del Estado Zulia, Venezuela) 1995;23(2): 125-151.

23. Di Prisco MC, Hagel I, Lynch NR, Barrios RM, Alvarez N, Lopez RI. Possible relationship be- tween allergic disease and infection by Giardia lamblia. Ann Allergy 1992;70:210.

Manuscrito recibido el 12 de octubre de 1999 y aceptado para publicación, tras revisión, el 10 de julio de 2000.

ANEXO 1. Indicadores antropométricos del estado nutricional de niños de 6 meses a 5 años de edad, según los parámetros propuestos por O'Donnell et al. ${ }^{\mathrm{a}}$

\begin{tabular}{|c|c|c|c|c|}
\hline Parámetro & $\begin{array}{l}\text { Deficiencia } \\
\text { grave }\end{array}$ & $\begin{array}{l}\text { Deficiencia } \\
\text { moderada }\end{array}$ & Deficiencia leve & Adecuado \\
\hline $\begin{array}{l}\text { Peso para talla } \\
\text { Puntaje Z } \\
\text { Percentil }\end{array}$ & $\begin{array}{c}<-3,0 \\
<-1\end{array}$ & $\begin{array}{c}-3,0 \text { a }-2,1 \\
1 \text { a } 2\end{array}$ & $\begin{array}{c}-2,0 \text { a }-1,1 \\
3 \text { a } 15\end{array}$ & $\begin{array}{c}-1,0 \text { a }+1,0 \\
16 \text { a } 84\end{array}$ \\
\hline $\begin{array}{l}\text { Talla para edad } \\
\text { Puntaje Z } \\
\text { Percentil }\end{array}$ & $\begin{array}{c}<-3,0 \\
<-1\end{array}$ & $\begin{array}{c}-3,0 \text { a }-2,1 \\
1 \text { a } 2\end{array}$ & $\begin{array}{c}-2,0 \text { a }-1,1 \\
3 \text { a } 15\end{array}$ & $\begin{array}{c}-1,0 \\
16\end{array}$ \\
\hline $\begin{array}{l}\text { Peso para edad } \\
\text { Puntaje Z } \\
\text { Percentil } \\
6 \text { a } 36 \text { meses } \\
4 \text { y } 5 \text { años }\end{array}$ & $\begin{array}{l}<-3,0 \\
<-1 \\
<70 \\
<65\end{array}$ & $\begin{array}{c}-3,0 \text { a }-2,1 \\
1 \text { a } 2 \\
70 \text { a } 79 \\
65 \text { a } 74\end{array}$ & $\begin{array}{c}-2,0 \text { a }-1,1 \\
3 \text { a } 14 \\
80 \text { a } 89 \\
75 \text { a } 89\end{array}$ & $\begin{array}{c}-1,0 \\
15 \\
90 \\
90\end{array}$ \\
\hline
\end{tabular}

ABSTRACT We investigated the influence of nutritional status, as determined from anthropometric measurement, and of helminthic infections on the immune response of children of low socioeconomic status in two rural communities in Venezuela: El Cardón in the

Influence of helminthic infections and nutritional status on the immune response of Venezuelan children state of Nueva Esparta and San Daniel in the state of Miranda. A total of 125 boys and girls between 2 and 15 years old participated in the study. Their socioeconomic stratum was determined by a modified Graffar method. A physical examination was performed, as was also an anthropometric evaluation that took into account three indicators-weight-for-height, weight-for-age, and height-for-age-according to parameters established by the World Health Organization. Other examinations included feces, secretory IgA in saliva, total serum IgE, and anti-Ascaris-specific immunoglobulins. The children in both of the communities were in strata IV and V of the of Graffar scale, with a significantly greater number of stratum V inhabitants in San Daniel $(P<0.001)$. The results suggest that exposure level and individual susceptibility to the parasites are determining factors in parasitic infection and immune system behavior. The intensity of the parasitic burden plays an important role in stimulating polyclonal IgE, which diminishes the effectiveness of the specific response to those infections. On the other hand, nutritional deficiencies could change the immune mechanisms of the mucous membranes, negatively influence the synthesis of secretory $\operatorname{IgA}$, and stimulate the production of polyclonal IgE. Poor sanitary and socioeconomic conditions promote more exposure to gastrointestinal parasites and a deficient nutritional status, which modulates the immune response and affects serum IgE and secretory IgA production mechanisms. 